\title{
Effet sur la stabilité des barrages en terre homogènes d'une variation des principaux paramètres
}

\author{
Effect of a variation in the main parameters \\ on the stability of homogeneous earth dams
}

\author{
E. ALONSO \\ Ingénieur, CEMAGREF* \\ T. BERNEDE \\ Ingénieur, CARA** \\ P. MORLIER \\ Professeur, Université Bordeaux I***
}

Rev. Franç. Géotech. n 63, pp. 23-37 (auril 1993)

\section{Résumé}

L'influence respective de chaque caractéristique mécanique et de chaque type de profil sur la stabilité des barrages en terre homogènes est présentée. Les valeurs prises en considération correspondent à celles qui ont été déterminées lors de l'étude de trente-cinq barrages. Les résultats obtenus permettent au projeteur de discerner les paramètres importants. Ils montrent aussi qu une même valeur du coefficient de sécurité n'a pas la même signification pour tous les talus. II est proposé une nouvelle approche du calcul de stabilité à long terme de ces ouvrages.

\section{Abstract}

There is a presentation of the respective influence of each mechanical characteristic and each type of slope on the stability of homogeneous earth dams. The values taken into account correspond to those which were determined during the study of thirty five dams. The results obtained enable the designer to ascertain the main parameters. They also show that the same factor of safety must not be applied to all of the slopes. A new approach to long-term stability analysis of these dams is hereby proposed.

\footnotetext{
50, avenue de Verdun, 33611 Gazinet Cedex.

*.48, rue Raymond-Lavigne, 33492 Le Bouscat Cedex.

*.. IUT A, 33405 Talence Cedex.
} 
Les barrages homogènes représentent quantitativement l'essentiel des barrages en terre réalisés en France. Leur construction continue à se développer en raison du fort accroissement des besoins en eau.

Ils sont constitués d'un matériau argileux qui assure à la fois les fonctions d'étanchéité et de stabilité, à la différence des barrages dits zonés pour lesquels ces fonctions sont assurées par des matériaux différents, ce qui permet d'obtenir une meilleure stabilité. La conséquence de cette structure homogène est leur hauteur relativement modeste, au maximum quelques dizaines de mètres, et aussi une certaine vulnérabilité due à la difficulté de bien connaître le comportement des matériaux fins.

Afin de mieux appréhender les conséquences d'une variation de chacun des principaux paramètres, mécaniques et géométriques, sur le coefficient de sécurité F qui caractérise la stabilité de ces ouvrages homogènes, nous avons effectué de nombreux calculs à partir de la structure de barrage simplifiée. Il ne s'agit pas ici d'une étude théorique mais d'une approche empirique basée sur des variations finies des différentes caractéristiques, l'éventail des valeurs considérées provenant de trentecinq barrages étudiés dans le Sud-Ouest de la France dont la hauteur par rapport au terrain naturel est comprise entre $4 \mathrm{~m}$ et $36 \mathrm{~m}$, l'ouvrage le plus important étant celui de MONTBEL dans le département de l'Ariège (1).

\section{LES DONNÉES PRISES EN COMPTE}

Elles concernent la géométrie des ouvrages, les caractéristiques des matériaux et les logiciels utilisés (2).

\subsection{Géométrie des ouvrages}

La figure 1 représente la coupe simplifiée adoptée pour les calculs. La ligne de saturation du talus amont est considérée horizontale (même après une vidange rapide) et sont négligées la protection amont en enrochements, la clé d'étanchéité et l'épaisseur du drain vertical (8).

La figure 2 montre la profondeur réelle du substratum résistant pour les trente-cinq sites étudiés, une purge partielle ou totale des alluvions étant jugée nécessaire pour une dizaine de sites, notamment ceux concernés

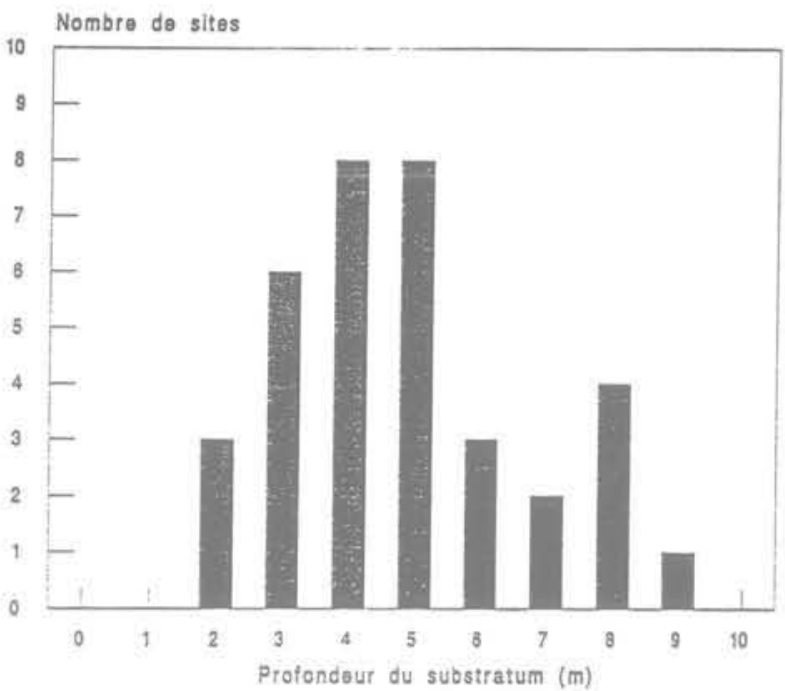

Fig. 2. - Profondeur du substratum pour les 35 sites.

Fig. 2. - Depth of substratum for the 35 sites.

par les plus grands ouvrages. Dans les calculs présentés ici, la fondation meuble est supposée homogène avec en général les trois épaisseurs suivantes: 0,5 et $10 \mathrm{~m}$. Les caractéristiques des remblais prises en compte sont regroupées dans le tableau 1 . La largeur en crête L correspond approximativement à la formule $\mathrm{L}=$ $3,6 \mathrm{H}^{1 / 3}-3$ où $\mathrm{H}$ est la hauteur du remblai exprimée en $\mathrm{m}$. Tableau 1. - Caractéristiques géométriques
des remblais prises en compte.

\begin{tabular}{|c|c|c|}
\hline $\begin{array}{c}\text { Hauteur H } \\
\text { du remblai } \\
(\mathrm{m})\end{array}$ & $\begin{array}{c}\text { Largeur crête L } \mathrm{L} \\
(\mathrm{m})\end{array}$ & $\begin{array}{c}\text { Dénivellation } \\
\text { niveau normal } \\
\text { des eaux-crête } \\
(\mathrm{m})\end{array}$ \\
\hline 10 & 4 & 2 \\
15 & 5 & 3 \\
20 & 6 & 3 \\
25 & 7 & 3 \\
30 & 8 & 3 \\
40 & 9 & 4 \\
50 & 10 & 4 \\
\hline
\end{tabular}

AVAL

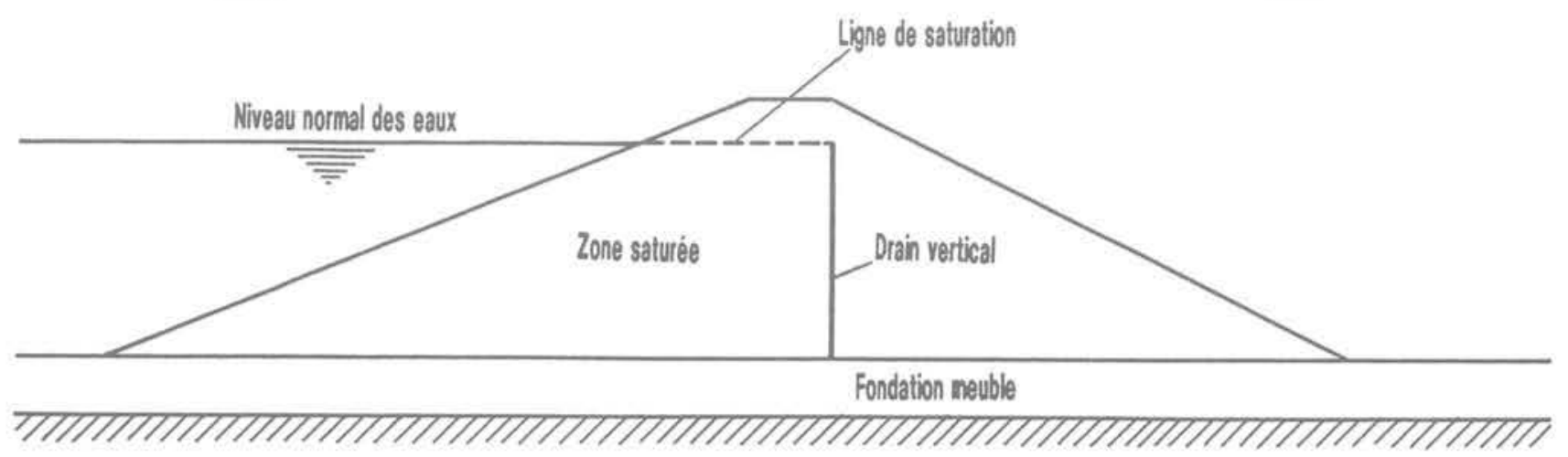

\section{Substratum}

Fig. 1. - Coupe simplifiée adoptée pour les calculs.

Fig. 1. - Simplified cross-section used for calculations. 
L'influence du profil des talus est déterminée dans les cas suivants :

- pente uniforme, changement de pente, risberme, pour la stabilité à long terme ;

— banquette pour la stabilité à court terme.

\subsection{Caractéristiques des matériaux}

\subsubsection{Valeurs obtenues lors de l'étude des trente-cinq sites}

Dans les trente-cinq sites étudiés, où prédominent les terrains molassiques, 134 échantillons intacts et remaniés prélevés par le CEMAGREF ont été soumis à des essais triaxiaux (critère de rupture $\sigma 1-\sigma 3$ maximum avec $\sigma 3$ variant généralement de 50 à $400 \mathrm{kPa}$ ). Tous ces échantillons, sauf 5 , contiennent plus de $40 \%$ d'éléments inférieurs à $80 \mu$, l'indice de plasticité WL - WP étant compris entre 10 et 40 (sauf une valeur à 55). Les échantillons remaniés des emprunts soumis aux essais triaxiaux sont préalablement compactés à l'énergie Proctor Normal et le plus souvent à leur teneur en eau naturelle. Le poids volumique à l'état initial des éprouvettes est compris entre 18 et $22 \mathrm{kN} / \mathrm{m}^{3}$ pour 131 échantillons, les 3 autres ayant une valeur inférieure à $18 \mathrm{kN} / \mathrm{m}^{3}$ (prélevés en fondation).

La répartition des caractéristiques obtenues lors des 125 essais triaxiaux consolidés non drainés effectués se trouve dans le tableau 2 (les faibles et les fortes valeurs de $\sigma 3$ donnent dans l'ensemble des cercles de rupture alignés bien qu'elles encadrent la contrainte de préconsolidation).

Bien que la tendance générale soit plutôt une évolution contraire de $c^{\prime}$ de o $^{\prime \prime \prime}$ il y a des couples de valeurs faibles et de valeurs fortes. Ces derniers sont proches des valeurs minimales $\left(c^{\prime}=10 \mathrm{kPa}\right.$ et $\left.\varphi^{\prime}=20^{\circ}\right)$ et maximales $\left(c^{\prime}=30 \mathrm{kPa}\right.$ et $\left.\varphi^{\prime}=30^{\circ}\right)$ prises en compte par la suite.

En ce qui concerne les échantillons intacts aucune différence n'a été constatée entre les valeurs de la cohésion $c^{\prime}$ des échantillons superficiels prélevés à moins de $2 \mathrm{~m}$ de profondeur (deux tiers des prélèvements) où l'action des cycles dessiccation-humidification entraîne une certaine surconsolidation et celles des échantillons prélevés entre $2 \mathrm{~m}$ et $5 \mathrm{~m}$ de profondeur (un tiers des prélèvements). Finalement très peu d'essais ont donné une cohésion $c^{\prime}$ de la fondation meuble inférieure à $10 \mathrm{kPa}$, ce qui signifie théoriquement que très peu de matériaux étaient normalement consolidés.

La répartition des valeurs de c obtenues lors des essais triaxiaux non consolidés non drainés effectués sur 28 échantillons intacts prélevés dans les fondations meubles se trouve dans le tableau 3 . Les valeurs de 0 sont en général nulles (matériau pratiquement saturé) ou bien égales à quelques degrés.

La répartition des valeurs de $c_{u}$ et $\varphi_{u}$ obtenues lors des essais triaxiaux non consolidés non drainés effectués sur 55 échantillons remaniés prélevés dans les emprunts se trouve dans le tableau 4 (lorsque l'enveloppe de rupture

Tableau 2. - Répartition des caractéristiques obtenues lors des triaxiaux consolidés non drainés.

\begin{tabular}{|l|c|c|c|c|c|c|}
\hline $\begin{array}{c}\text { Caractéristiques } \\
\text { Echantillons }\end{array}$ & $5 \leqslant c^{\prime}<10 \mathrm{kPa}$ & $10 \leqslant c^{\prime} \leqslant 30 \mathrm{kPa}$ & $30<c^{\prime} \leqslant 40 \mathrm{kPa}$ & $10^{\circ}<\varphi^{\prime} \leqslant 20^{\circ}$ & $20^{\circ} \leqslant \varphi^{\prime} \leqslant 30^{\circ}$ & $30^{\circ}<\varphi^{\prime}<40^{\circ}$ \\
\hline $\begin{array}{l}36 \text { échantillons intacts } \\
\text { des fondations }\end{array}$ & $11 \%$ & $86 \%$ & $3 \%$ & $11 \%$ & $75 \%$ & $14 \%$ \\
\hline $\begin{array}{l}89 \text { échantillons } \\
\text { compactés (emprunts) }\end{array}$ & $5,5 \%$ & $87,5 \%$ & $7 \%$ & $4,5 \%$ & $75,5 \%$ & $20 \%$ \\
\hline
\end{tabular}

Tableau 3. - Répartition des valeurs de $c_{y}$ en kPa des fondations meubles (variation de l'indice de consistance entre parenthèses).

\begin{tabular}{|c|c|c|c|c|}
\hline $5<\mathrm{c}_{u}<25$ & $25 \leqslant \mathrm{c}_{u}<50$ & $50 \leqslant \mathrm{c}_{u}<75$ & $75 \leqslant \mathrm{c}_{\mathrm{u}}<100$ & $100<\mathrm{c}_{u}<125$ \\
\hline $\begin{array}{c}7 \% \\
\left(0,5<\mathrm{I}_{\mathrm{c}}<0,7\right)\end{array}$ & $\left(0,5<\mathrm{I}_{\mathrm{c}}<1,1\right)$ & $\left(0,8<\mathrm{I}_{\mathrm{c}}<1,1\right)$ & $\left(0,8<\mathrm{I}_{\mathrm{c}}<1,1\right)$ & $\left(1<\mathrm{I}_{\mathrm{c}}<1,1\right)$ \\
\hline
\end{tabular}

Tableau 4. - Répartition des valeurs de $c_{\mathrm{u}}$ et $\varphi$ des emprunts compactés.

\begin{tabular}{|c|c|c|c|c|c|c|}
\hline Valeurs de $\varphi_{u}$ & \multicolumn{4}{|c|}{$\varphi_{\mathrm{u}}=0$ ou $<10^{\circ}$} & \multicolumn{2}{|c|}{$10^{\circ} \leqslant \varphi_{u} \leqslant 2020^{\circ}<\varphi_{u}<35^{\circ}$} \\
\hline Valeur de $c_{u}$ en $\mathrm{kPa}$ & $25<\mathrm{C}_{u}<50$ & $50 \leqslant c_{u}<75$ & $75 \leqslant c_{u}<100$ & $100 \leqslant c_{u} \leqslant 150$ & $100 \leqslant c_{u}<150$ & $25 \leqslant c_{u}<100$ \\
\hline $\begin{array}{l}\text { Pourcentage } \\
\text { d'échantillons }\end{array}$ & $3,5 \%$ & $18 \%$ & $16,5 \%$ & $31 \%$ & $20 \%$ & $11 \%$ \\
\hline $\begin{array}{l}\text { Teneur en eau initiale } \\
\text { par rapport à l'OPN }\end{array}$ & $\begin{array}{l}\mathrm{OPN}_{\mathrm{a}}+4 \\
\mathrm{OPN}=5\end{array}$ & $\begin{array}{l}\mathrm{OPN}+2 \\
\stackrel{\mathrm{a}}{\mathrm{OPN}}+4\end{array}$ & $\begin{array}{c}\mathrm{OPN} \\
\stackrel{a}{a} \\
O P N\end{array}$ & $\begin{array}{l}\mathrm{OPN}-2 \\
\mathrm{à} \\
\mathrm{OPN}+1\end{array}$ & $\begin{array}{l}\text { OPN - } 4 \\
\text { à } \\
\text { OPN ? }\end{array}$ & $\begin{array}{c}\text { OPN }-5 \\
\text { à } \\
\text { OPN - } 3\end{array}$ \\
\hline $\mathrm{I}_{\mathrm{c}}$ & 1 & 1 à 1,2 & 1,1 à 1,3 & 1,2 à 1,4 & 1,3 à 1,5 & 1,4 à 1,6 \\
\hline
\end{tabular}


de Mohr a un changement de pente une droite moyenne est prise en compte). Comme ces valeurs dépendent surtout de l'humidité des matériaux compactés, l'écart entre la teneur en eau initiale des éprouvettes et l'Optimum Proctor Normal est précisé dans le tableau 4. A l'OPN, l'indice de consistance est compris entre 1,1 et 1,4 sauf pour quelques échantillons, notamment les plus micacés. La variation de l'in. dice de consistance est également mentionnée au tableau 4 en éliminant les échantillons dont I à l'OPN est supérieur à 1,4.

Signalons aussi qu'à l'essai œdométrique les contraintes de préconsolidation des matériaux se situent entre 70 et $250 \mathrm{kPa}$ pour les emprunts compactés à l'énergie Proctor Normal et entre 50 et $180 \mathrm{kPa}$ pour les fondations meubles, aucune corrélation précise n'apparaissant entre ces dernières valeurs et $c_{i}$.

\subsubsection{Valeurs prises en compte}

Elles correspondent aux valeurs précédentes.

Le poids volumique des matériaux de la fondation meuble et du remblai, saturés ou non saturés, est pris égal à $20 \mathrm{kN} / \mathrm{m}^{3}$.

Les caractéristiques mécaniques intrinsèques des matériaux après consolidation (contraintes effectives) pour les calculs de stabilité à long terme sont prises égales à :

- 10,20,30 kPa pour la cohésion $\mathrm{c}^{\prime}$;

- $20^{\circ}, 25^{\circ}, 30^{\circ}$ pour l'angle de frottement interne $\varphi^{\prime}$.

Les caractéristiques mécaniques des matériaux avant consolidation (contraintes totales) pour les calculs de stabilité à court terme sont prises égales à :

$-\mathrm{c}_{\mathrm{u}}=20$ à $100 \mathrm{kPa}$ et $\varphi_{\mathrm{u}}=0$ pour la fondation; - $\mathrm{c}_{\mathrm{u}}=50$ à $100 \mathrm{kPa}$ et $\varphi_{\mathrm{u}}=0$ à $30^{\circ}$ pour le remblai.

Ces caractéristiques mécaniques, à court terme et à long terme, permettent d'obtenir des pentes convenables pour les petits et moyens barrages mais, par contre, la réalisation de pentes très douces est nécessaire pour les barrages homogènes dont la hauteur atteint plusieurs dizaines de mètres (l'influence des forces de cohésion diminue lorsque la hauteur augmente).

La stabilité à court terme des remblais constitués de matériaux argileux humides (teneur en eau supérieure à l'Optimum Proctor Normal) est aussi traitée à l'aide des caractéristiques mécaniques intrinsèques et de la pression interstitielle de construction évaluée par l'expression $r_{u} \cdot \gamma \cdot h$ où $r_{u}$ est un coefficient compris entre 0 et 1 et oû́ $\gamma$.h représente la contrainte verticale totale due au poids dela colonne de terre située au-dessus du point considéré (paragraphe 4.3.).

\subsection{Les logiciels utilisés}

Ils sont basés sur des méthodes de calcul (3) à la rupture (équilibre limite). Il s'agit des logiciels suivants;

- logiciel STAB (CEMAGREF) basé sur la méthode de FELLENIUS, utilisé pour la plupart des calculs;
- logiciel STAB R (Virginia Polytechnic Institute and State University) basé sur la méthode de BISHOP simplifié (10) (composante verticale des forces intertranches négligée) utilisé surtout pour les calculs relatifs aux pressions interstitielles (définies par le coefficient $r_{u}$ );

- logiciel SLOPE 8R (Virginia Polytechnic Institute and State University) basé sur la méthode de SPEN. CER, qui considère non seulement l'équilibre des moments mais aussi celui des forces, utilisé pour les ruptures non circulaires (11).

Quelques calculs de ruptures non circulaires ont également été réalisés à l'aide du logiciel NIXES et TROLLS basé sur la méthode des perturbations (4).

Toutes ces méthodes reviennent à affecter la cohésion et le frottement du même coefficient de sécurité $F$.

Avec la méthode de FELLENIUS les forces de cisaillement pour l'ensemble des tranches sont égales à $\Sigma c^{\prime}, 1 / F+\Sigma N^{\prime} \cdot \operatorname{tg} \varphi^{\prime} / F$. Avec les méthodes de BISHOP et de SPENCER la force de cisaillement à la base d'une tranche est égale à $c^{\prime} \cdot 1 / F+N^{\prime} \cdot \operatorname{tg} \varphi^{\prime} / F$ :

$1=$ longueur de la base de la tranche;

$N^{\prime}$ = force normale effective s'exerçant sur la base de la tranche.

Avec la méthode des perturbations où le massif n'est pas découpé en tranches mais considéré globalement, la contrainte de cisaillement est égale à $c^{\prime} / F+\sigma^{\prime} \cdot \operatorname{tg} \varphi^{\prime} / F$, $\sigma^{\prime}$ étant la contrainte normale effective.

\section{INFLUENCE RESPECTIVE DES CARACTÉRISTIQUES MÉCANIQUES INTRINSÈQUES DES MATÉRIAUX}

Les talus considérés ici n'ont ni risberme, ni banquette, ni changement de pente. Les hauteurs de remblai considérées sont $10,15,20,25,30$ et $40 \mathrm{~m}$. Les calculs sont effectués à l'aide du logiciel STAB (FELLENIUS).

Les calculs de stabilité en rupture circulaire avec les autres méthodes entraîneraient pour $\mathrm{F}$ des différences de faible ampleur, de l'ordre de 0,1, la méthode de FELLENIUS étant généralement la plus pessimiste (5).

\subsection{Cas de la stabilité du talus amont à long terme}

Après consolidation des matériaux la stabilité du talus amont est calculée avec les caractéristiques intrinsèques lors d'une vidange rapide de la retenue,

On considère une épaisseur de fondation meuble et une hauteur de remblai et on détermine avec les caractéristiques moyennes (cohésion de $20 \mathrm{kPa}$ et angle de frottement interne de $25^{\circ}$ pour la fondation et le remblai) la pente du talus permettant d'obtenir un coefficient de sécurité $F$ voisin de 1,5. On étudie ensuite l'effet sur F d'une variation de la cohésion de la fondation $\left(\Delta c^{\prime}=\right.$ $10 \mathrm{kPa}$ ), de l'angle de frottement interne de la fondation $\left(\Delta \varphi^{\prime}=5^{\circ}\right)$, de la cohésion du remblai $\left(\Delta c^{\prime}=10 \mathrm{kPa}\right)$ et de l'angle de frottement interne du remblai $\left(\Delta \varphi^{\prime}=\right.$ $\left.5^{\circ}\right)$, ce qui entraîne 81 calculs $\left(3^{4}\right)$ de stabilité. On 
constate que la variation d'une caractéristique $(10 \mathrm{kPa}$ ou $5^{\circ}$ ) entraine une variation relativement constante de $\mathrm{F}$ (écart maximum de quelques centièmes) quelle que soit la valeur initiale et quelles que soient les trois autres caractéristiques. $\Delta \mathrm{F}$ est donc pratiquement proportionnel à $\Delta c^{\prime}$ et $\Delta \varphi^{\prime}$ avec une précision toutefois variable. Lorsque le cercle critique ne coupe pas la fondation (plus résistante) on est ramené au cas limite de la fondation meuble d'épaisseur nulle (lorsqu'une purge est nécessaire, le remblai et la fondation ont les mêmes caractéristiques mécaniques et le cercle critique passe dans cette dernière). Les courbes de la figure 3 permettent d'évaluer pour chaque épaisseur de fondation meuble et chaque hauteur de barrage, l'influence respective de chacune des quatre caractéristiques mécaniques sur le coefficient de sécurité $F$. Les points des courbes correspondent à un $\Delta \mathrm{F}$ moyen, les valeurs extrêmes se trouvant généralement à + ou $-0,02$ à 0,03 .

Par exemple pour un barrage de $10 \mathrm{~m}$ sur une fondation meuble d'épaisseur $5 \mathrm{~m}$ une diminution de $5^{\circ}$ de $\varphi^{\prime}$ remblai fait baisser $F$ de 0,04 tandis qu'une diminution de $10 \mathrm{kPa}$ de la cohésion de la fondation entraîne une chute de 0,27 ; par contre pour une hauteur de 30 à $35 \mathrm{~m}$ les deux $\Delta \mathrm{F}$ sont proches de 0,10 . Toujours pour une fondation de $5 \mathrm{~m}$, on constate que la variation des deux autres caractéristiques provoque quelle que soit la hauteur du barrage, un $\Delta \mathrm{F}$ sensiblement constant, approximativement égal à 0,10 pour une variation de $10 \mathrm{kPa}$ de la cohésion du remblai et à 0,15 pour une variation de $5^{\circ}$ de $\varphi^{\prime}$ fondation.

Avec une épaisseur de la fondation de $10 \mathrm{~m}$, l'influence relative des caractéristiques du remblai diminue. Une variation de $5^{\circ}$ de $\varphi^{\prime}$ entraîne un $\Delta \mathrm{F}$ proche de 0,20 s'il s'agit de la fondation, et voisin de 0,05 s'il s'agit du remblai.

Lorsque la fondation n'est pas concernée par le cercle critique on observe que jusqu'à une hauteur de $30 \mathrm{~m}$ une variation de la cohésion de $10 \mathrm{kPa}$ a plus d'influence sur $F$ qu'une variation de $\varphi^{\prime}$ de $5^{\circ}$; pour $\Delta \mathrm{c}^{\prime}=10 \mathrm{kPa}, \Delta \mathrm{F}$ atteint 0,55 pour un barrage d'une dizaine de mètres.

\subsection{Cas de la stabilité du talus aval à long terme}

En ce qui concerne la stabilité du talus aval, on constate sur la figure 4 que l'influence de $\varphi^{\prime}$ est plus importante que précédemment (ce qui est logique, le talus aval n'étant pas saturé).

Pour une fondation meuble d'épaisseur 5 à $10 \mathrm{~m}$ une variation de $5^{\circ}$ de $\varphi^{\prime}$ fondation entraine un $\Delta F$ compris entre 0,20 et 0,30 .

Lorsque la fondation n'est pas impliquée par le cercle critique, $\Delta \mathrm{F}$ est voisin de 0,40 pour une variation de $5^{\circ}$ de $\varphi^{\prime}$ remblai et $\Delta F$ est supérieur à 0,50 pour $\Delta c^{\prime}=10 \mathrm{kPa}$ lorsque le remblai a une hauteur de $10 \mathrm{~m}$.

\subsection{Conséquences sur le coefficient de sécurité}

L'intérêt pratique des courbes des figures 3 et 4 est de montrer l'importance respective de chaque caractéris-
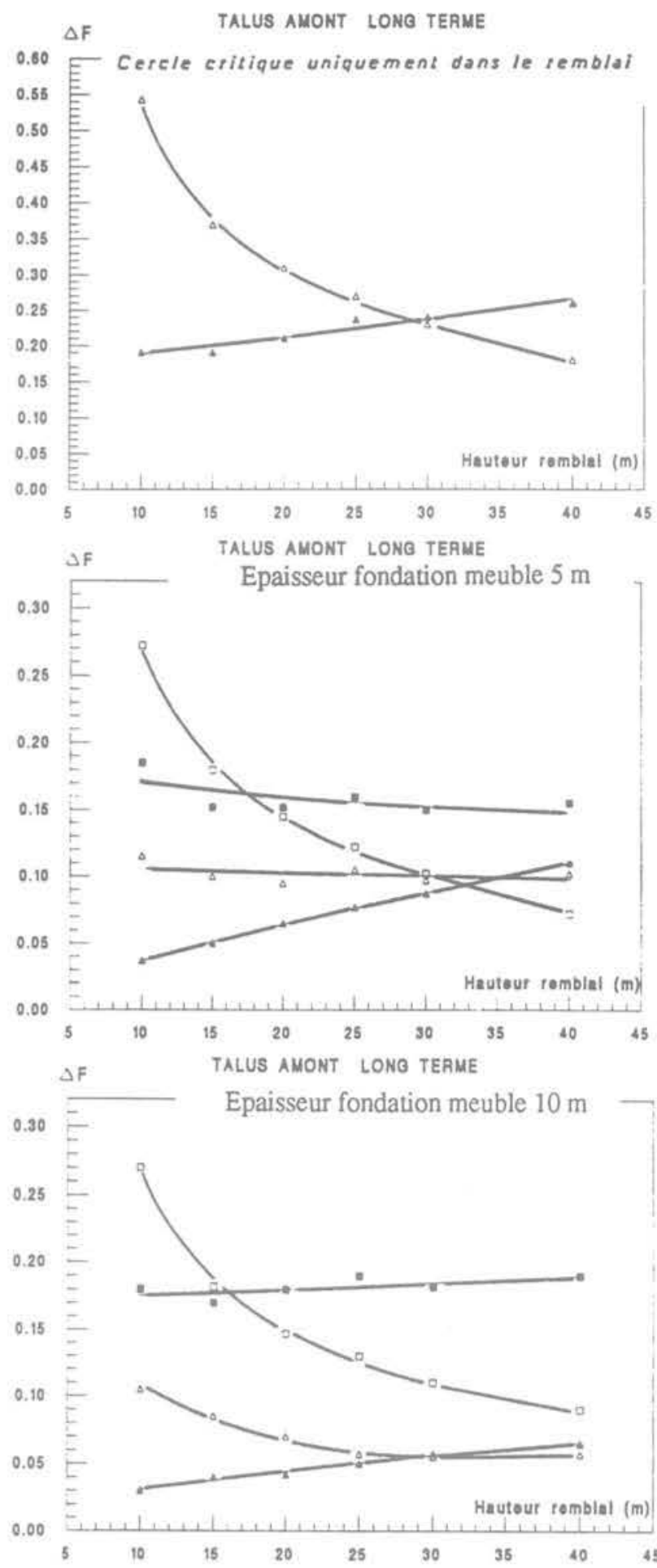

$\square$ Fondation: $\Delta \mathrm{C}^{\prime}=10 \mathrm{kPa}$

- Fondation: $\Delta \Phi^{\prime}=5^{\circ}$

$\triangle$ Remblai : $\Delta \mathrm{C}^{\prime}=10 \mathrm{kPa}$

$\Delta$ Remblai : $\Delta \Phi^{\prime}=5^{\circ}$

Fig. 3. - Influence de chaque caractéristique mécanique sur la stabilité à long terme du talus amont.

Fig. 3. - Influence of each mechanical characteristic on the long-term stability of the upstream slope.

tique mécanique intrinsèque sur la stabilité du talus considéré. Ces courbes facilitent le choix des valeurs à prendre en compte dans les calculs de stabilité. 

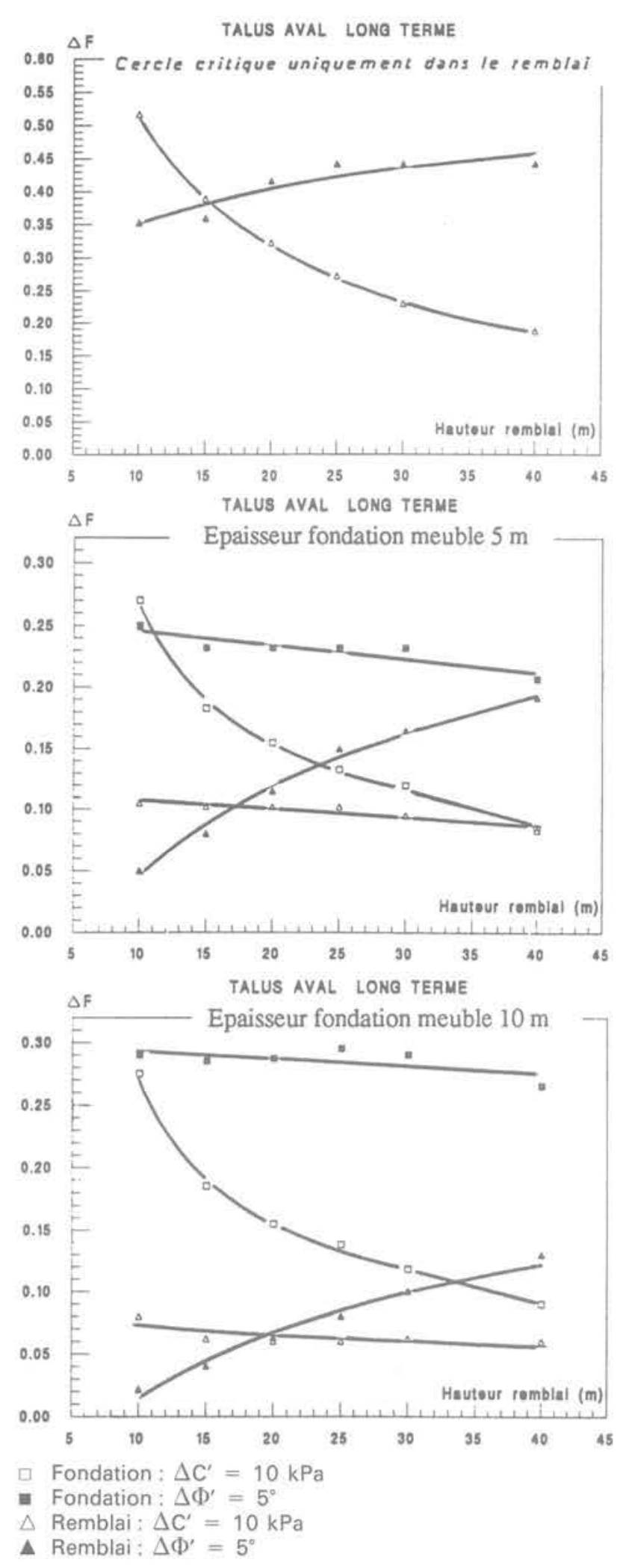

Fig. 4. - Influence de chaque caractéristique mécanique sur la stabilité à long terme du talus aval.

Fig. 4. - Influence of each mechanical characteristic on the long-terme stability on the downstream slope.

Il convient d'être particulièrement prudent d'une part avec les caractéristiques prises en compte pour la fondation meuble (hétérogénéités, difficultés du prélève. ment des échantillons intacts, $\Delta \mathrm{F}$ généralement supérieurs à ceux dus aux variations des caractéristiques du remblai) et d'autre part avec la cohésion des matériaux intacts ou compactés (fonction notamment de la surconsolidation) qui est un paramètre difficile à déterminer avec précision alors que son rôle est important même s'il diminue avec la hauteur du barrage. Il est à noter que l'interprétation des essais triaxiaux conduit à faire évoluer c' et $\varphi^{\prime}$ en sens contraire et qu'une erreur de $10 \mathrm{kPa}$ est plus probable qu'une erreur de $5^{\circ}$.

Le coefficient de sécurité $\mathrm{F}$ ne doit pas être pris systématiquement égal à 1,5 mais adapté au cas traité (7). Dans les courbes précédentes (fig. 3 et 4 ), si l'on additionne les $\Delta \mathrm{F}$ dus aux variations de $10 \mathrm{kPa}$ et de $5^{\circ}$ correspondant à une épaisseur de fondation meuble et à une hauteur de remblai (précision de 0,1 environ suivant les caractéristiques initiales et la pente du talus), on a la valeur la plus faible $\Sigma \Delta \mathrm{F}=0,4$ pour un talus amont en vidange rapide avec une fondation meuble de $10 \mathrm{~m}$ et une hauteur de remblai de $40 \mathrm{~m}$; la valeur la plus élevée est $\Sigma \Delta \mathrm{F}=0,9$ pour un talus aval avec fondation résistante (cercle critique uniquement dans le remblai) et de hauteur $10 \mathrm{~m}$. Nous proposons de choisir comme coefficient de sécurité $1+\Sigma \Delta \mathrm{F}$ ce qui donne, suivant le cas considéré, $\mathrm{F}$ compris entre 1,4 et 1,9 pour une sécurité équivalente. En effet, un coefficient de sécurité correspondant à une même chute des caractéristiques mécaniques des matériaux (fondation et remblai ou remblai seulement) de $10 \mathrm{kPa}$ et de $5^{\circ}$ est plus élevé en général de 0,1 à 0,2 :

- pour le talus aval que pour le talus amont;

- pour une fondation non affectée par le cercle critique que pour une fondation meuble d'épaisseur $10 \mathrm{~m}$ qui l'est;

- pour un petit barrage de hauteur $10 \mathrm{~m}$ que pour un grand barrage de $40 \mathrm{~m}$.

Si l'on effectue les calculs de stabilité d'un ouvrage de hauteur inférieure à $10 \mathrm{~m}$ il faut prendre un coefficient de sécurité élevé, supérieur à 2 dans certains cas.

Si l'on prend $c^{\prime}=0$ pour la fondation meuble $\Sigma \Delta F$ correspond aux trois autres caractéristiques, ce qui donne un coefficient de sécurité compris entre 1,3 et 1,5 pour des barrages homogènes de 10 à $40 \mathrm{~m}$ sur 5 à $10 \mathrm{~m}$ de fondation meuble.

Si l'on souhaite accroître la sécurité du talus aval (rupture retenue pleine) on peut ajouter à $1+\Sigma \Delta \mathrm{F}$ une valeur de 0,2 par exemple.

En pratique, une fois les caractéristiques mécaniques des matériaux déterminées on diminue la cohésion de $10 \mathrm{kPa}$ et l'angle de frottement interne de $5^{\circ}$ (fondation et remblai) et on vérifie que $\mathrm{F}$ est encore supérieur à 1 (ou 1,2 pour le talus aval si l'on souhaite une sécurité supplémentaire).

Rappelons qu'avec la méthode de FELLENIUS, si l'on obtient un coefficient de sécurité $F=1,5$ avec $c^{\prime}$ et $\varphi^{\prime}$, on a $F=1$ avec $c^{\prime} / 1,5$ et tg $\varphi^{\prime} / 1,5$, ce qui correspond d'une part à une diminution $\Delta \mathrm{c}^{\prime}$ de 3,3 à $10 \mathrm{kPa}$ pour des valeurs de c' de 10 à $30 \mathrm{kPa}$ et d'autre part à une diminution $\Delta \varphi^{\prime}$ de 6,4 à $9^{\circ}$ pour des valeurs de $\varphi^{\prime}$ de 20 à $30^{\circ}$. En prenant une sécurité correspon- 
dant à $\Delta \mathrm{c}^{\prime}=10 \mathrm{kPa}$ et $\Delta \varphi^{\prime}=5^{\circ}$ on est donc plus sévère pour la cohésion et moins pour l'angle de frottement interne, ce qui nous parait plus logique.

\section{INFLUENCE DU PROFIL DU TALUS SUR LA STABILITÉ A LONG TERME}

On détermine ici à l'aide du logiciel STAB l'influence respective de la pente uniforme du talus, du changement de pente et de la risberme.

\subsection{Influence de la pente uniforme du talus}

La pente est exprimée par le rapport $1 / \mathrm{x}$ où $\mathrm{x}$ est la projection horizontale du talus correspondant à une projection verticale égale à l'unité (fig. 6).

Tous les autres paramètres étant fixés lépaisseur de la fondation meuble, hauteur du remblai, caractéristiques mécaniques), si l'on fait varier $\mathrm{x}$, on obtient une relation pratiquement linéaire avec le coefficient de sécurité $F$. De plus, les droites $\mathrm{F}$ fonction de $\mathrm{x}$ relatives à chaque hauteur de remblai sont parallèles. On a donc représenté sur la figure 5 pour trois épaisseurs de fondation meuble la variation $\Delta \mathrm{F}$ fonction de $\Delta \mathrm{x}$ pour les caractéristiques mécaniques moyennes $c^{\prime}=20 \mathrm{kPa}$ et $\varphi^{\prime}=25^{\circ}$ (fondation et remblai). Les résultats obtenus avec les autres caractéristiques mécaniques sont sensiblement équivalents.

Une réduction de la pente du talus (accroissement de $\Delta \mathrm{x}$ ) entraîne une augmentation de $\mathrm{F}$ plus importante pour le talus aval et pour une épaisseur de fondation meuble plus fiable.

\subsection{Influence du changement de pente}

Le problème peut être traité à section de remblai constante ou à section de remblai croissante. Ces cas sont illustrés par la figure 6 .

\subsubsection{Section de remblai croissante}

F étant le coefficient de sécurité correspondant au talus initial de pente uniforme $1 / x$ il s'agit de déterminer le gain $\Delta \mathrm{F}$ dû à l'accroissement $\Delta \mathrm{x}=\mathrm{x}_{2}-\mathrm{x}_{1}$.
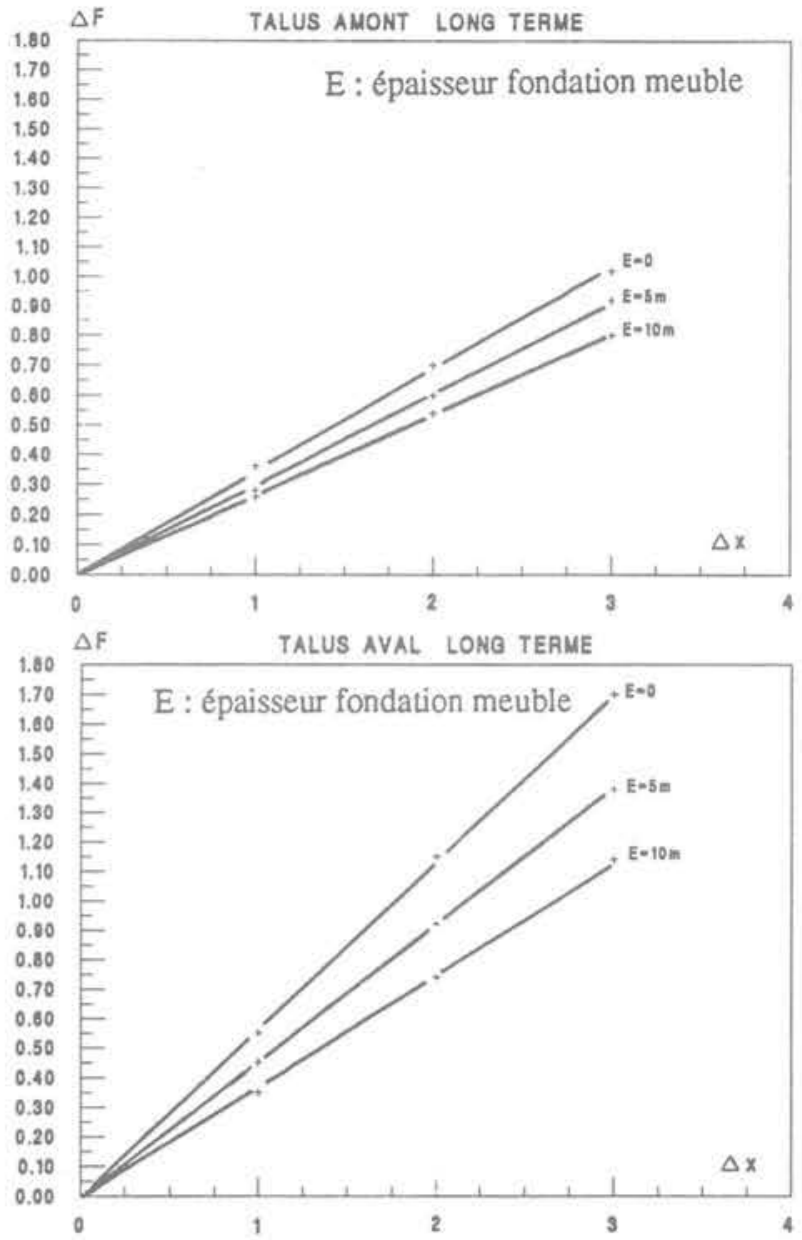

Fig. 5. $-\Delta F$ fonction de $\Delta x$ (pente uniforme) pour $c^{\prime}=20 \mathrm{kPa}$ et $\varphi^{\prime}=25^{\circ}$ (fondation et remblai).

Fig. 5. - $\Delta F$ as a function of $\Delta x$ (uniform slope) for $c^{\prime}=20 \mathrm{kPa}$ and $\varphi^{\prime}=25^{\circ}$ (foundation and fill).

La figure 7 concerne les résultats obtenus avec les caractéristiques mécaniques moyennes $c^{\prime}=20 \mathrm{kPa}$ et $\varphi^{\prime}=25^{\circ}$ (fondation et remblai) pour une fondation meuble d'épaisseur $5 \mathrm{~m}$ et pour des hauteurs $\mathrm{H}$ de remblai de 15,20 et $30 \mathrm{~m}$, avec pour les deux dernières hauteurs le cas aussi de deux changements de pente, l'un à $\mathrm{H} / 3$ et l'autre à $2 / 3 \mathrm{H}$. $\Delta x$ correspondant alors à la somme des deux réductions de pente (qui sont
Section croissonte

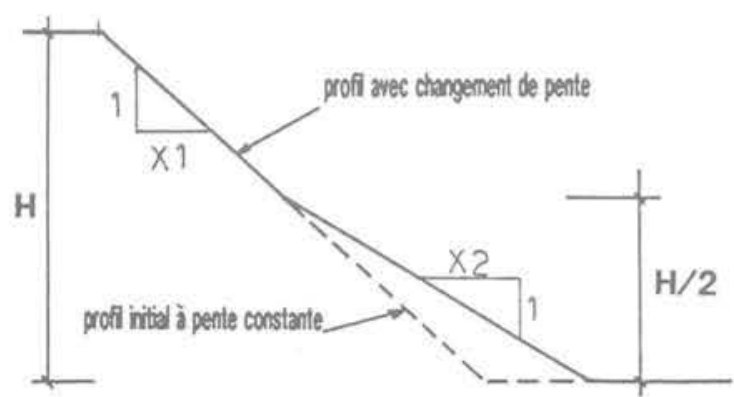

Section constante

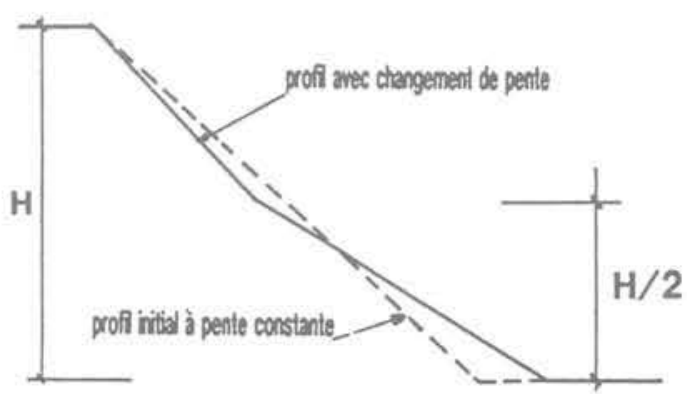

Fig. 6. - Profils avec changement de pente à mi-hauteur.

Fig. 6. - Slopes with mid-height change of slope. 

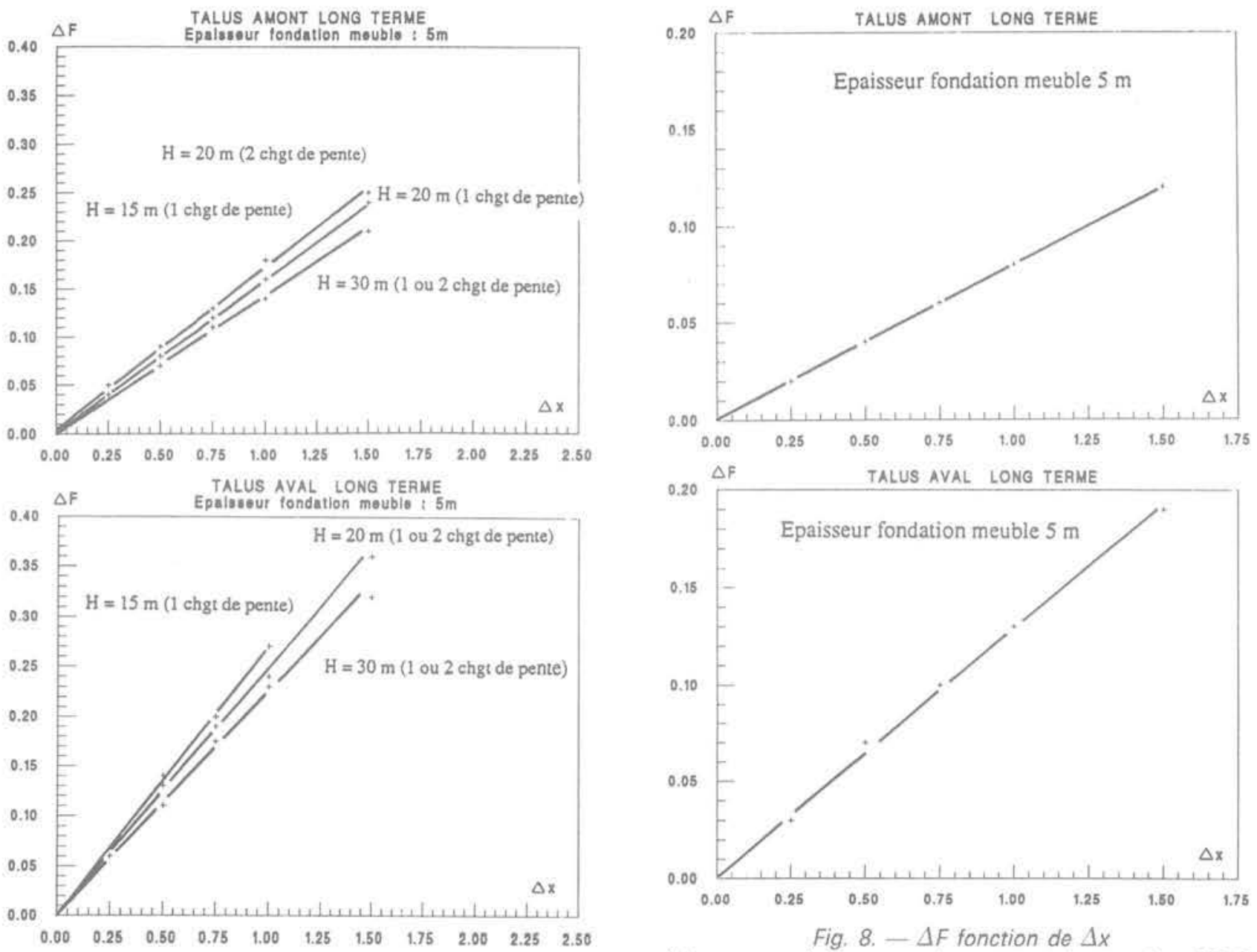

Fig. 8. $-\Delta F$ fonction de $\Delta x$

Fig. 7. $-\Delta F$ fonction de $\Delta x$

(changement de pente, section croissante) pour $c^{\prime}=20 \mathrm{kPa}$ et $\phi^{\prime}=25^{\circ}$ (fondation et remblai) et pour une épaisseur de fondation meuble de $5 \mathrm{~m}$.

Fig. 7. $-\Delta F$ as a function of $\Delta x$

(change of slope, incleasing cross-section) for $C^{\prime}=20 \mathrm{kPa}$ and $\varphi^{\prime}=25^{\circ}$ (foundation and fill) and for a $5 \mathrm{~m}$ thickness of loose foundation.

prises égales). Les différences entre un et deux changements de pente sont négligeables et la hauteur du remblai a peu d'influence sur $\Delta \mathrm{f}$.

Avec les autres épaisseurs de fondation meuble et les autres caractéristiques mécaniques on obtient des résultats sensiblement éviqualents.

Un changement de pente à mi-hauteur correspondant à une valeur de $\Delta \mathrm{x}$ égale au double de celle relative à la réduction de pente uniforme entraîne la même po. sition pour le pied du talus, un $\Delta \mathrm{F}$ du même ordre (voir fig. 5 et 7) mais un volume supplémentaire de matériaux moindre. Le changement de pente est donc une solution intéressante à condition toutefois que la partie supérieure du talus puisse être plus raide.

\subsubsection{Section de remblai constante}

Un seul changement de pente à mi-hauteur est pris en compte et les valeurs de $\mathrm{x}_{1}$ et de $\mathrm{x}_{2}$ sont telles que la section du remblai reste constante. La figure 8 repré-

(changement de pente, section constante) pour $c^{\prime}=20 \mathrm{kPa}$ et $\varphi^{\prime}=25^{\circ}$ (fondation et remblai) et pour une épaisseur de fondation meuble de $5 \mathrm{~m}$.

Fig. 8. $-\Delta F$ as a function of $\Delta x$

(change of slope. constant cross-section) for $c^{\prime}=20 \mathrm{kPa}$ and $\varphi^{\prime}=25^{\circ}$ (foundation and fill) and for a $5 \mathrm{~m}$ thickness of loose foundation.

sente $\Delta \mathrm{F}$ fonction de $\Delta \mathrm{x}=\mathrm{x}_{2}-\mathrm{x}_{1}$ avec les caractéristiques mécaniques moyennes $c^{\prime}=20 \mathrm{kPa}$ et $\phi^{\prime}$ $=25^{\circ}$ (fondation et remblai) et une épaisseur de $5 \mathrm{~m}$ pour la fondation meuble. L'influence de la hauteur du remblai est négligeable. Les autres épaisseurs de fondation meuble et les autres caractéristiques mécaniques donnent des résultats sensiblement équivalents.

Par rapport au cas précédent (section croissante) une même valeur de $\Delta \mathrm{x}$ donne approximativement un $\Delta \mathrm{F}$ deux fois moins élevé mais il n'y a pas d'augmentation du volume du remblai. Cette solution est intéressante si la partie supérieure du talus peut être raidie.

\subsection{Influence de la risberme}

Comme précédemment le problème peut être traité à section de remblai constante ou à section croissante. La figure 9 illustre les différents cas. L'influence sur $\mathrm{F}$ de la largeur L de la risberme est déterminée pour les hauteurs de remblai de $15,20,30$ et $40 \mathrm{~m}$, les deux dernières hauteurs étant concernées aussi par la double risberme (chacune de largeur $\mathrm{L} / 2$ ). 


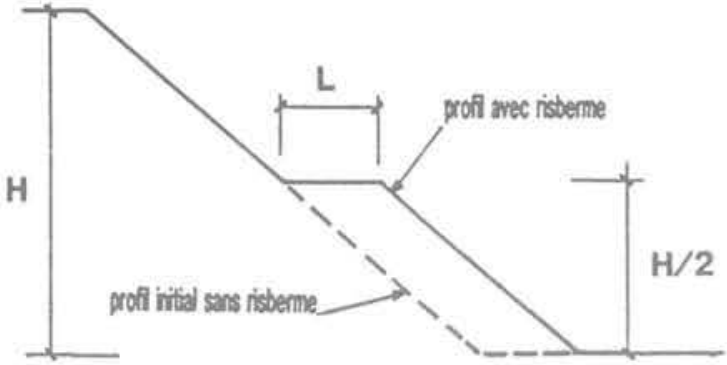

profil à risberme unique

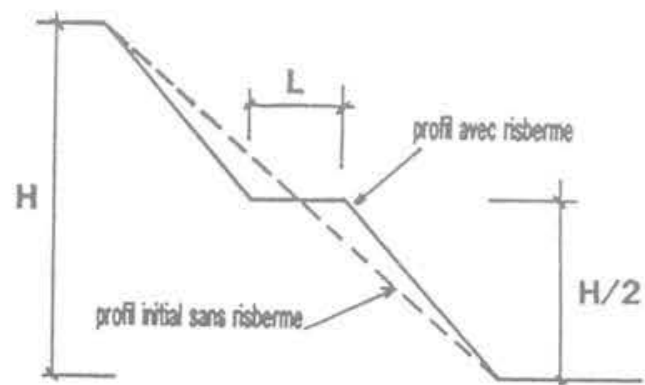

profil à risberme unique

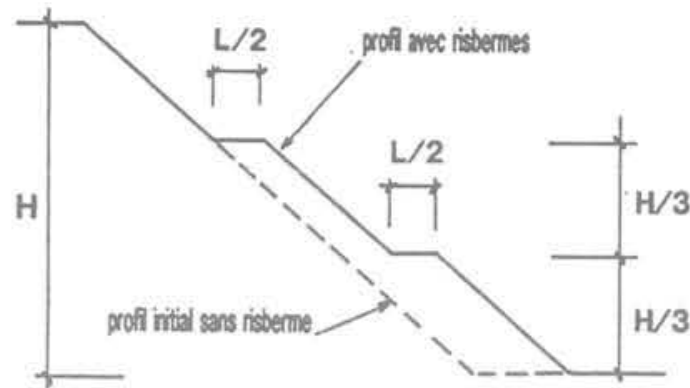

profil à double risberme

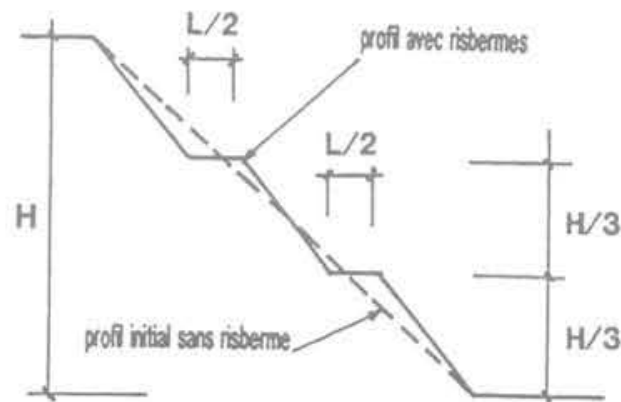

profil à double risberme

Fig. 9. - Profils avec une ou deux risbermes (section croissante et section constante).

Fig. 9. - Slopes wiht one or two berms (increasing section and constant section).

La pente du talus est la même au-dessus et au-dessous d'une risberme.

\subsubsection{Section de remblai croissante}

Les résultats concernant le gain $\Delta \mathrm{F}$ en fonction de la largeur de risberme pour les trois épaisseurs de fondation meuble (dont l'influence n'est pas négligeable) et pour les caractéristiques mécaniques moyennes $c^{\prime}$ $=20 \mathrm{kPa}$ et $\varphi^{\prime}=25^{\circ}$ (fondation et remblai) se trouvent sur la figure 10. Avec les autres caractéristiques mécaniques on obtient des résultats sensiblement équivalents.

La double risberme est moins favorable que la risberme unique à mi-hauteur (la risberme haute a plus un rôle moteur que stabilisateur) mais elle facilite la maitrise des eaux de ruissellement et l'entretien du parement.

Le rôle stabilisateur de la risberme est important et une même largeur $\mathrm{L}$ entraîne un accroissement $\Delta \mathrm{F}$ plus élevé :

- pour le talus aval;

- pour les faibles épaisseurs de fondation meuble;

- pour les petits barrages.

Une risberme de largeur égale à la moitié de la hauteur du remblai entraîne un gain $\Delta \mathrm{F}$ analogue à celui dû à une réduction de pente uniforme correspondant à $\Delta x$ $=1$ (voir fig. 5) pour un volume supplémentaire de matériaux moins important. La risberme est donc une solution intéressante si la pente du talus peut être suffisamment raide.

\subsubsection{Section de remblai constante}

A section constante la mise en place d'une risberme entraine par rapport au profil initial, une augmentation de la pente. Si cela est possible le gain $\Delta \mathrm{F}$ obtenu, proportionnel à la largeur de risberme L, est d'environ 30 à $50 \%$ du $\Delta \mathrm{F}$ précédent (risberme avec section croissante) mais le volume du remblai n'est pas accru. La figure 11 représente le cas où les caractéristiques mécaniques sont $c^{\prime}=20 \mathrm{kPa}$ et $\varphi^{\prime}=25^{\circ}$ (fondation et remblai). Des résultats sensiblement équivalents sont obtenus avec les autres caractéristiques mécaniques.

\section{STABILITÉ A COURT TERME}

En fin de travaux, avant le remplissage de la retenue et avant toute consolidation des matériaux, les calculs de stabilité du barrage concernent le talus le plus raide ou les deux talus.

Outre la rupture circulaire, le cas de la rupture non circulaire est également à traiter (c'est évident lorsqu'il y a une couche mince molle). Si la fondation meuble peu résistante est épaisse le calcul en rupture par poin. çonnement peut fournir un premier résultat approximatif (6).

Nous allons ici déterminer l'influence sur le coefficient de sécurité $F$ des caractéristiques mécaniques $c_{u}$ et $\varphi_{0}$ lors de la rupture circulaire d'un talus de pente uni- 

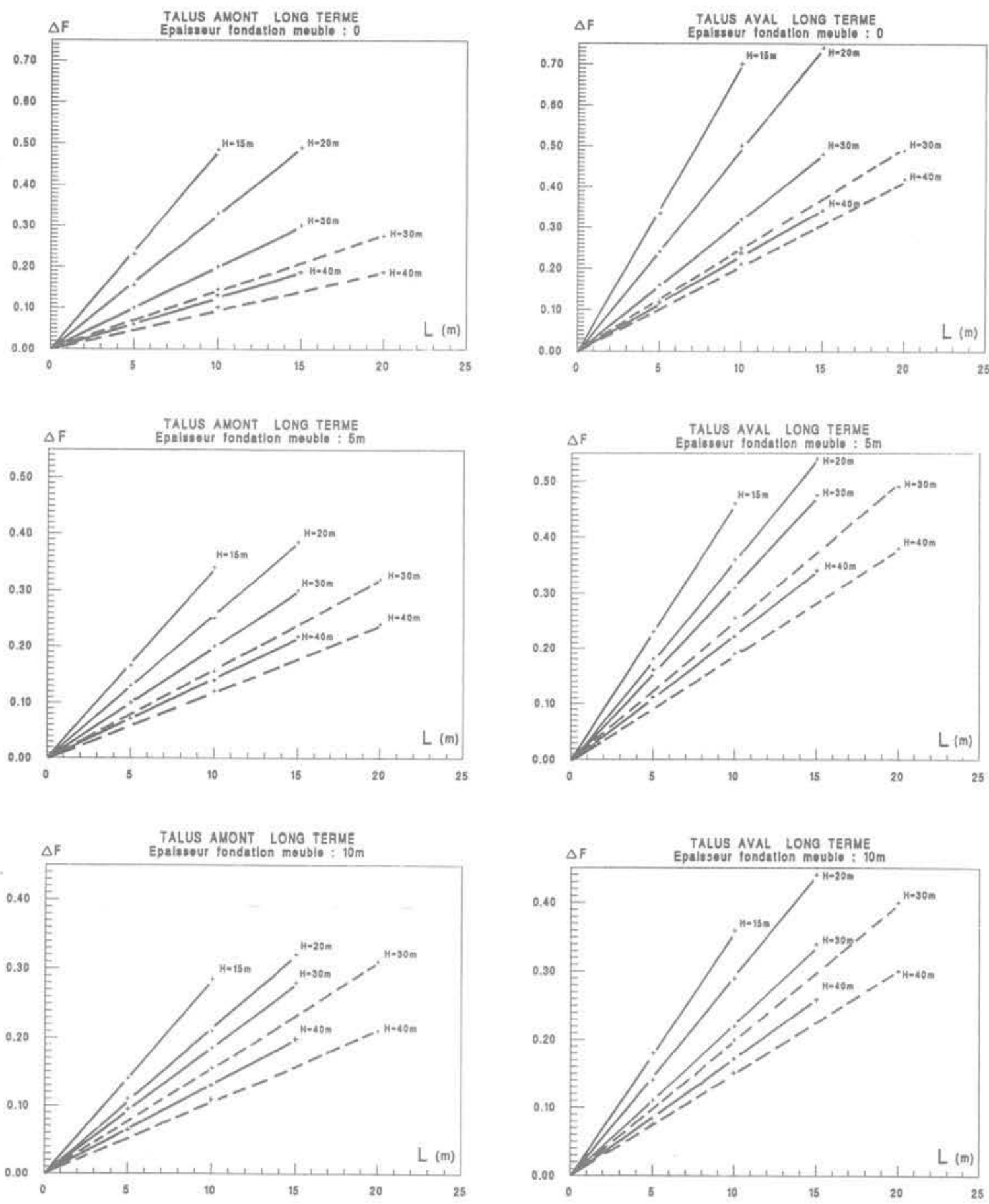

: profil à risberme unique $(\mathrm{H} / 2)$

- - : profil à double risberme $(\mathrm{H} / 3$ et $2 \mathrm{H} / 3)$

Fig. 10, - Augmentation du coefficient de sécurité due aux risbermes, le volume de remblai étant croisant ( $c^{\prime}$ et $\varphi^{\prime}$ fondation et remblai : $20 \mathrm{kPa}$ et $25^{\circ}$ ).

Fig. 10. - Increase in factor of safety due of the berms. will fill volume increasing ( $C^{\prime}$ and $\varphi^{\prime}$ foundation and fill: $20 \mathrm{kPa}$ and $25^{\circ}$ ).

forme et l'influence sur F de la largeur de la banquette stabilisatrice mise en place pour éviter une purge des matériaux peu résistants de la fondation. La fin de ce paragraphe 4 est consacrée au cas particulier des pressions interstitielles de construction se développant dans un remblai constitué de matériaux argileux humides (9). 

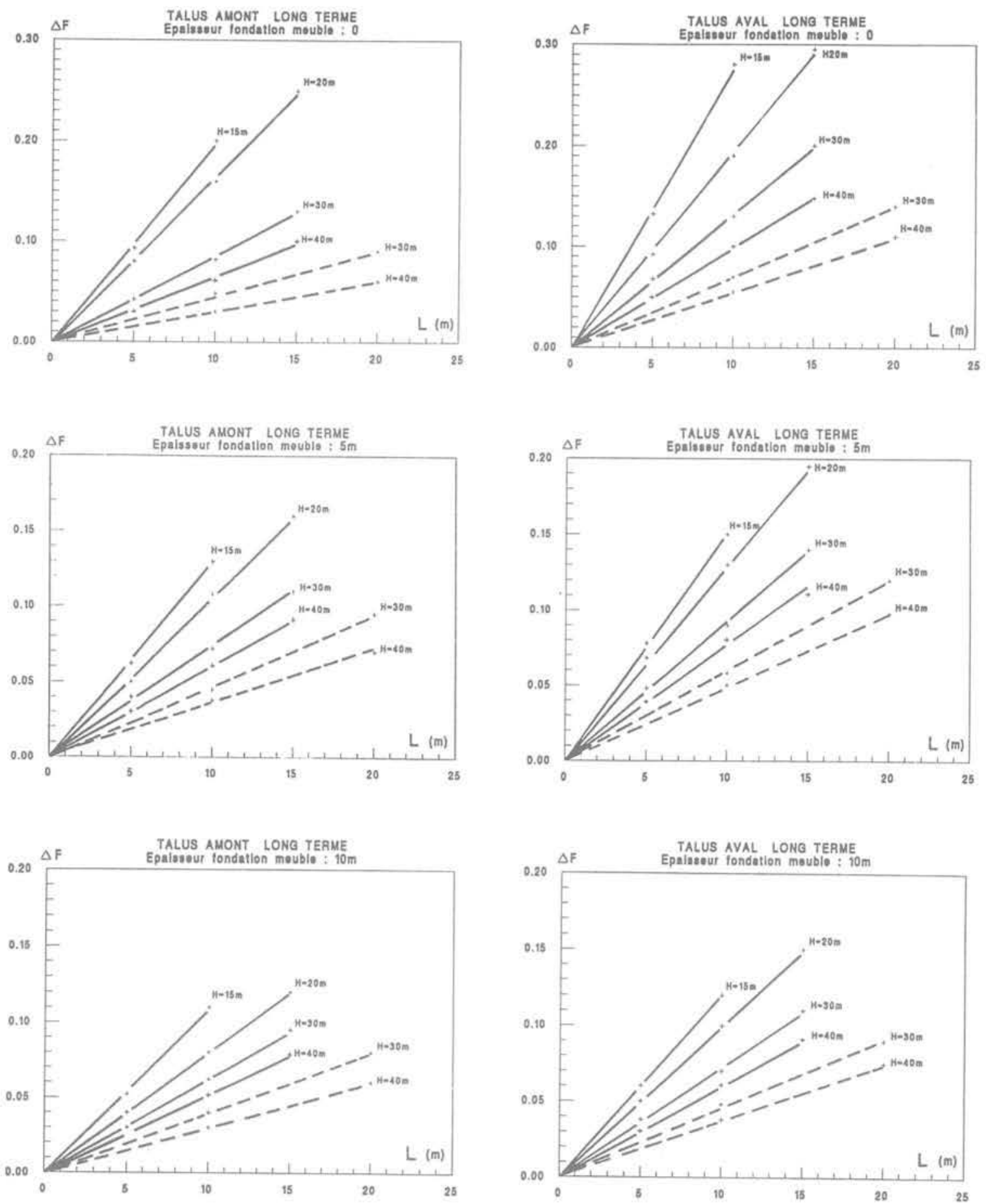

— : profil à risberme unique $(\mathrm{H} / 2)$

$---\quad$ : profil à double risberme $(\mathrm{H} / 3$ et $2 \mathrm{H} / 3)$

Fig. 11. - Augmentation du coefficient de sécurité due aux risbermes, le volume de remblai étant maintenu constant ( $c^{\prime}$ et $\varphi^{\prime}$ fondation et remblai: $20 \mathrm{kPa}$ et $25^{\circ}$ ),

Fig. 11. - Increase in factor of safety due of the berms, with fill remaining constant $\left(\mathrm{c}^{\prime}\right.$ and $\varphi^{\prime}$ foundation and fill: $20 \mathrm{kPa}$ and $25^{\circ}$ ). 


\subsection{Influence des caractéristiques mécaniques lors d'une rupture circulaire}

Les caractéristiques mécaniques prises en compte sont les suivantes:

- pour la fondation meuble:

$$
\begin{aligned}
& \text { - } c_{u}=20,40,60,80 \text { et } 100 \mathrm{kPa} \\
& \text { soit } \Delta c_{u}=20 \mathrm{kPa} \text {; } \\
& \text { - } \varphi_{u}=0 \text {; } \\
& \text { - pour le remblai : } \\
& \text { - } c_{u}=50,75 \text { et } 100 \mathrm{kPa} \\
& \text { soit } \Delta c_{u}=25 \mathrm{kPa} ; \\
& \text { - } \varphi_{u}=0,10,20 \text { et } 30^{\circ} \\
& \text { soit } \Delta \varphi_{u}=10^{\circ} \text {. }
\end{aligned}
$$

En opérant comme au paragraphe 2 on obtient l'influence respective de chacune des trois caractéristiques sur le coefficient de sécurité F. L'ensemble des résultats se trouve sur la figure 12 . Deux cas sont représentés: fonction meuble d'épaisseur $5 \mathrm{~m}$ et $10 \mathrm{~m}$.

Une diminution de $\mathrm{c}_{\mathrm{y}}$ égale à $20 \mathrm{kPa}$ pour une fondation meuble d'épaisseur 5 à $10 \mathrm{~m}$ entraine une chute de $\mathrm{F}$ de 0,55 lorsque la hauteur du barrage est d'une dizaine de mètres; cette chute est réduite de moitié lorsque la hauteur est de $20 \mathrm{~m}$.

Une variation de c du remblai égale à $25 \mathrm{kPa}$ provoque une variation sensiblement constante de $F$ quelle que soit la hauteur du barrage, d'environ 0,23 si l'épaisseur de la fondation est de $5 \mathrm{~m}$ et de 0,13 si l'épaisseur est de $10 \mathrm{~m}$.
Si l'on considère la stabilité du remblai seul (fondation résistante, purge) et uniquement une variation de 25 $\mathrm{kPa}$ de la cohésion non drainée, $\varphi_{\|}$étant nul, on obtient les résultats suivants (tableau 5 ):

$\Delta \mathrm{F}$ est plus élevé pour les barrages de hauteur plus modeste et aussi pour les pentes plus douces.

\subsection{Influence de la largeur de la banquette stabilisatrice}

Lorsque la cohésion non drainée $c_{y}$ de la fondation meuble est faible il $y$ a en pratique deux solutions:

- la purge des matériaux dont le coût n'est pas excessif si l'épaisseur est relativement faible, par exemple inférieure au cinquième de la hauteur du barrage;

- la mise en place de banquettes stabilisatrices (dont l'inconvénient est toutefois d'allonger l'ouvrage de restitution) qui peuvent permettre d'accroitre suffisamment $F$.

La solution consistant à mettre en place un dispositif drainant dans la fondation pour accélérer sa consolidation est exceptionnelle.

La hauteur de la banquette est ici prise égale à la moitié de la hauteur du remblai.

La figure 13 montre l'évolution du coefficient de sécurité $\mathrm{F}$ en fonction de la largeur $\mathrm{L}$ de la banquette pour trois épaisseurs de fondation meuble $3 \mathrm{~m}, 5 \mathrm{~m}$ et $10 \mathrm{~m}$ et pour les cinq cas représentés dans le tableau 6 où la cohésion non drainée de la fondation meuble est faible par rapport à la hauteur du barrage et où le remblai a une cohésion inférieure au double de celle


Fig. 12. - Influence de chaque caractéristique mécanique sur la stabilité à court terme.

Fig. 12. - Influence of each mechanical characteristic on short-term stability.

Tableau 5. - Valeurs de $\Delta F$ correspondant à $\Delta c_{u}=25 \mathrm{kPa}$.

\begin{tabular}{|c|c|c|c|c|c|}
\hline Hauteur du remblai & $15 \mathrm{~m}$ & $20 \mathrm{~m}$ & $25 \mathrm{~m}$ & $30 \mathrm{~m}$ & $40 \mathrm{~m}$ \\
\hline $\begin{array}{c}\text { Pentes usuelles } \\
\text { considérées }\end{array}$ & $1 / 2$ à $1 / 3,5$ & $1 / 2,5$ à $1 / 4$ & $1 / 2,5$ à $1 / 4$ & $1 / 2,5$ à $1 / 4,5$ & $1 / 3$ à $1 / 5$ \\
\hline$\Delta \mathrm{F}$ & 0,68 à 0,94 & 0,57 à 0,77 & 0,46 à 0,61 & 0,38 à 0,55 & 0,32 à 0,44 \\
\hline
\end{tabular}



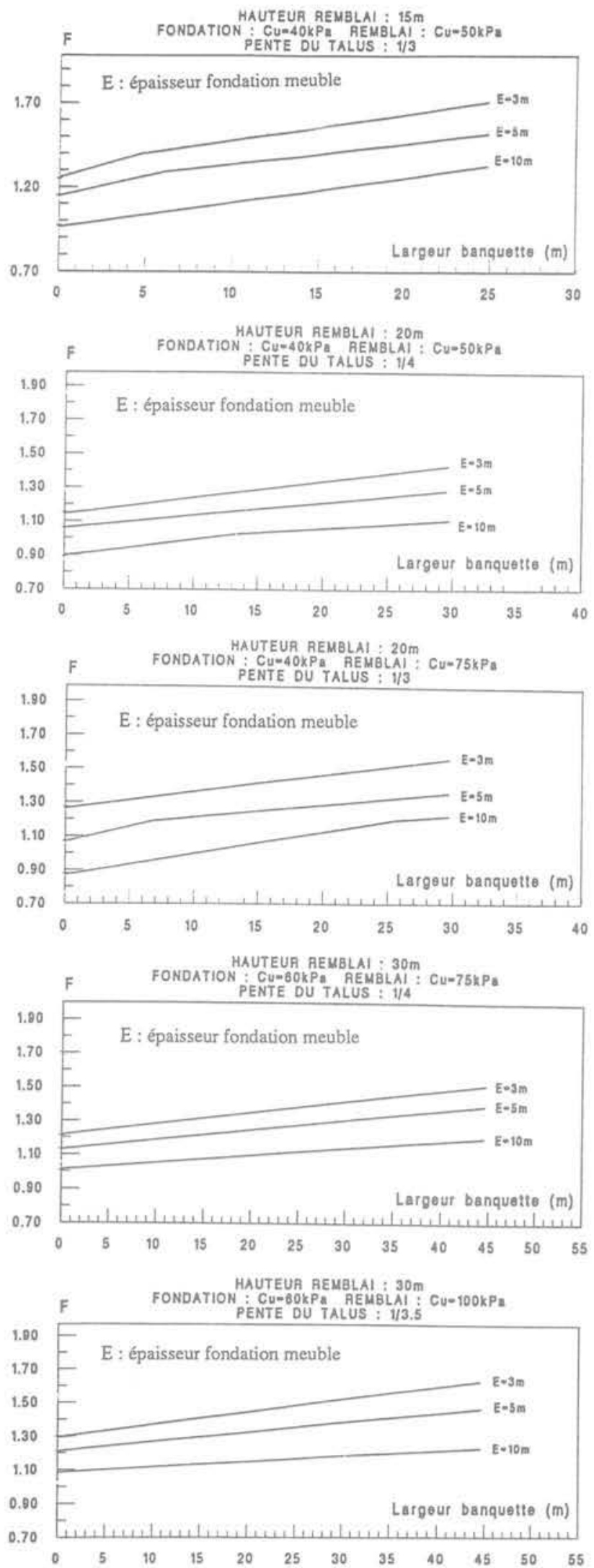

Fig. 13. - F fonction de la largeur de la banquette pour les cinq cas considérés.

Fig. 13, - F as a function of the width of the stabilizing berm for the five examples under consideration.

de la fondation $(\varphi=0$ pour la fondation et pour le remblai). Les calculs de stabilité effectués concernent les ruptures circulaire et non circulaire.

La méthode de BISHOP et utilisée pour la rupture circulaire et celle de SPENCER pour la rupture non circulaire en considérant une surface de glissement horizontale à la base de la fondation meuble.

Les droites $\mathrm{F}$ fonction de $\mathrm{L}$ représentant la stabilité au glissement concernent le calcul qui donne la valeur de F la plus faible. Les droites qui n'ont pas de changement de pente sont relatives à des glissements non circulaires et pour celles qui ont un changement de pente, c'est la partie située à gauche qui représente le glissement circulaire.

Pour améliorer sensiblement $F$ il faut une largeur de banquette importante, l'objectif à court terme étant d'atteindre un coefficient de sécurité d'au moins 1,3 à 1,5 suivant le cas considéré.

\subsection{Influence des pressions interstitielles de construction}

Les incidents survenus pendant ou peu après la construction de quelques barrages homogènes constitués de matériaux fins, argileux et humides, compactés au moins partiellement à l'aide de rouleaux vibrants lisses à une énergie permettant d'obtenir la densité la plus élevée possible (et donc un degré de saturation supérieur à $90 \%$ ) n'ont pu être expliqués par les caractéristiques mécaniques déterminées en contraintes totales (cohésion non drainée $c_{\mathrm{u}}$ relativement élevée).

Le développement de pressions interstitielles de construction élevées a été confirmé par quelques cellules. Notons également que le feuilletage observé et la mauvaise liaison probable entre certaines couches posent aussi le problème de la valeur réelle des caractéristiques mécaniques.

Le but des calculs réalisés ici à l'aide du logiciel STAB $\mathrm{R}$ (BISHOP) est de rechercher la valeur de $r_{u}$ critique qui, associée aux caractéristiques intrinsèques ' $c^{\prime}$ et $\varphi^{\prime}$, donne un coefficient de sécurité $\mathrm{F}$ égal à 1 lorsque la pente a été préalablement déterminée avec le logiciel STAB pour un $F$ voisin de 1,5 .

Pour simplifier, nous considérons que le remblai repose directement sur une fondation résistante (cercle critique uniquement dans le remblai) et que le coefficient $r_{4}$ est constant dans tout le massif dont la pente est uniforme.

Les trois cas suivants sont traités:

- talus aval dont la pente est définie par la cohésion non drainée $c_{u}$ (on prend $\varphi_{u}=0$ );

- talus aval dont la pente est définie par le calcul à long terme ;

- talus amont dont la pente est définie par le calcul à long terme (vidange rapide).

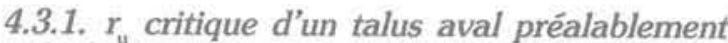 calculé à court terme}

Les caractéristiques des 14 talus considérés dont $\mathrm{F}=$ 1,50 à 1,58 à court terme se trouvent dans le tableau 7 (logiciel STAB). 
Tableau 6. - Valeurs prises en compte pour les calculs à court terme.

\begin{tabular}{|c|c|c|c|c|c|}
\hline Hauteur du remblai & $15 \mathrm{~m}$ & \multicolumn{2}{|c|}{$20 \mathrm{~m}$} & \multicolumn{2}{c|}{$30 \mathrm{~m}$} \\
\hline Pente du talus & $1 / 3$ & $1 / 4$ & $1 / 3$ & $1 / 4$ & $1 / 3,5$ \\
\hline $\mathrm{c}_{\mathrm{u}}$ en $\mathrm{kPa}:$ & & & & & \\
$\begin{array}{l}\text { fondation } \\
\text { remblai }\end{array}$ & 40 & 40 & 40 & 60 & 60 \\
\hline
\end{tabular}

Tableau 7. - Talus dont $F=1,50$ à 1,58 à court terme.

\begin{tabular}{|c|c|c|c|c|c|c|c|}
\hline \multicolumn{2}{|c|}{$\mathrm{H}=15 \mathrm{~m}$} & \multicolumn{2}{c|}{$\mathrm{H}=20 \mathrm{~m}$} & \multicolumn{2}{c|}{$\mathrm{H}=30 \mathrm{~m}$} & \multicolumn{2}{c|}{$\mathrm{H}=40 \mathrm{~m}$} \\
\hline $\mathrm{c}_{\mathrm{u}}$ en $\mathrm{kPa}$ & pente & $\mathrm{c}_{u}$ en $\mathrm{kPa}$ & pente & $\mathrm{c}_{u}$ en $\mathrm{kPa}$ & pente & $\mathrm{c}_{u}$ en $\mathrm{kPa}$ & pente \\
\hline 50 & $1 / 2,5$ & 50 & $1 / 4$ & 70 & $1 / 4,5$ & 90 & $1 / 4,75$ \\
\hline 60 & $1 / 2$ & 60 & $1 / 3$ & 80 & $1 / 3,5$ & 100 & $1 / 4$ \\
\hline & & 70 & $1 / 2,25$ & 90 & $1 / 3$ & 110 & $1 / 3,5$ \\
\hline & & 75 & $1 / 2$ & 100 & $1 / 2,5$ & 120 & $1 / 3$ \\
\hline
\end{tabular}

Les caractéristiques mécaniques intrinsèques suivantes représentant un large éventail de valeurs ont été prises en compte pour les calculs de stabilité à court terme associant $c^{\prime}$ et $\varphi^{\prime}$ à la pression interstitielle de construction $r_{\mathrm{r}} \cdot \gamma \cdot \mathrm{h}$ :

$-\mathrm{c}^{\prime}=10 \mathrm{kPa}$ et $\varphi^{\prime}=20^{\circ}$, valeurs faibles prises en compte seulement lorsqu'elles ne sont pas plus défavorables que $c_{u}$;

$-c^{\prime}=10 \mathrm{kPa}$ et $\varphi^{\prime}=30^{\circ}$, cohésion faible et angle élevé :

$-c^{\prime}=20 \mathrm{kPa}$ et $\varphi^{\prime}=25^{\circ}$, valeurs moyennes;

$-c^{\prime}=30 \mathrm{kPa}$ et $\varphi^{\prime}=20^{\circ}$, cohésion forte et angle peu élevé ;

- $\mathrm{c}^{\prime}=30 \mathrm{kPa}$ et $\varphi^{\prime}=30^{\circ}$, valeurs fortes

La figure 14 permet d'obtenir les valeurs de $r_{4}$ qui entraînent un coefficient de sécurité égal à 1 (méthode de BISHOP).

Plus $c_{u}$ est faible et $c^{\prime}$ et $\varphi^{\prime}$ sont forts (donc $c_{u}$ impose une pente bien plus douce que $c^{\prime}$ et $\sigma^{\prime}$ ne le feraient) plus $r_{u}$ doit être élevé, de 0,8 à plus de 0,9 , pour que la rupture intervienne. Par contre avec un $\mathrm{c}_{y}$ fort et un couple $c^{\prime}, \varphi^{\prime}$ qui ne l'est pas (donc $c_{\mathrm{u}}$ impose une pente à peine plus douce que $c^{\prime}$ et $\varphi^{\prime}$ ne le feraient), il suffit d'un $r_{u}$ de 0,4 et 0,5 , pour que la rupture intervienne.

Notons toutefois qu'un $c_{\text {u }}$ fort implique un matériau moins humide qu'un $c_{u}$ faible.

\subsection{2. $r_{u}$ critique d'un talus aval préalablement calculé à long terme}

Lorsque les caractéristiques intrinsèques $c^{\prime}$ et $\varphi^{\prime}$ définissent la pente du talus aval à long terme avec un coefficient de sécurité d'environ 1,6 , il suffit d'un $\mathrm{r}_{u}$ de l'ordre de 0,4 à 0,5 pour que la rupture intervienne en fin de construction. Ces résultats, conformes aux pré. cédents, mettent en évidence la vulnérabilité d'un talus aval constitué de matériaux argileux humides.

\subsection{3. $r_{u}$ critique d'un talus amont préalablement calculé à long terme}

En faisant varier la hauteur du remblai (sur fondation résistante) et les caractéristiques mécaniques intrin. sèques, les calculs effectués montrent que le coefficient de sécurité de 1,5 obtenu avec la méthode de FELLENIUS lors d'une vidange rapide implique un $r_{u}$ de 0,40 à 0,46 . La rupture intervient pour un $r_{u}$ compris entre 0,6 et 0,7 .

Il faut donc assurer une mise en place des matériaux n'entraînant pas un développement important des pressions interstitielles ou bien un dispositif drainant coûteux doit être prévu.

\section{CONCLUSION}

A partir d'une structure de barrage homogène simplifiée et de l'éventail des mesures concernant trente-cing sites étudiés dans le Sud-Ouest, les nombreux calculs de stabilité effectués montrent bien l'influence respective de chaque paramètre.

En fonction du talus considéré, amont ou aval, de la hauteur du barrage et de l'épaisseur de la fondation meuble, il est possible de déterminer à l'aide des abaques précédents avec une précision satisfaisante, l'effet sur la stabilité d'une variation d'une caractéristique mécanique ou d'une modification du profil.

Pour la stabilité à long terme, une fois les caractéristiques mécaniques déterminées et un profil adopté, nous proposons de diminuer la cohésion de $10 \mathrm{kPa}$ et l'angle de frottement interne de $5^{\circ}$ (fondation et rem. blai) et ensuite de vérifier que $F$ est supérieur ou égal à 1 pour le talut amont et à 1,2 pour le talus aval (afin d'obtenir une sécurité supplémentaire pour ce dernier). Des valeurs différentes de $10 \mathrm{kPa}$ et de $5^{\circ}$ peuvent aussi être retenues. Le changement de pente, la mise en place d'une risberme, permettent dans certains cas une réduction appréciable du volume du remblai.

Pour la stabilité à court terme, la réalisation de banquettes très larges est nécessaire pour améliorer sen. 

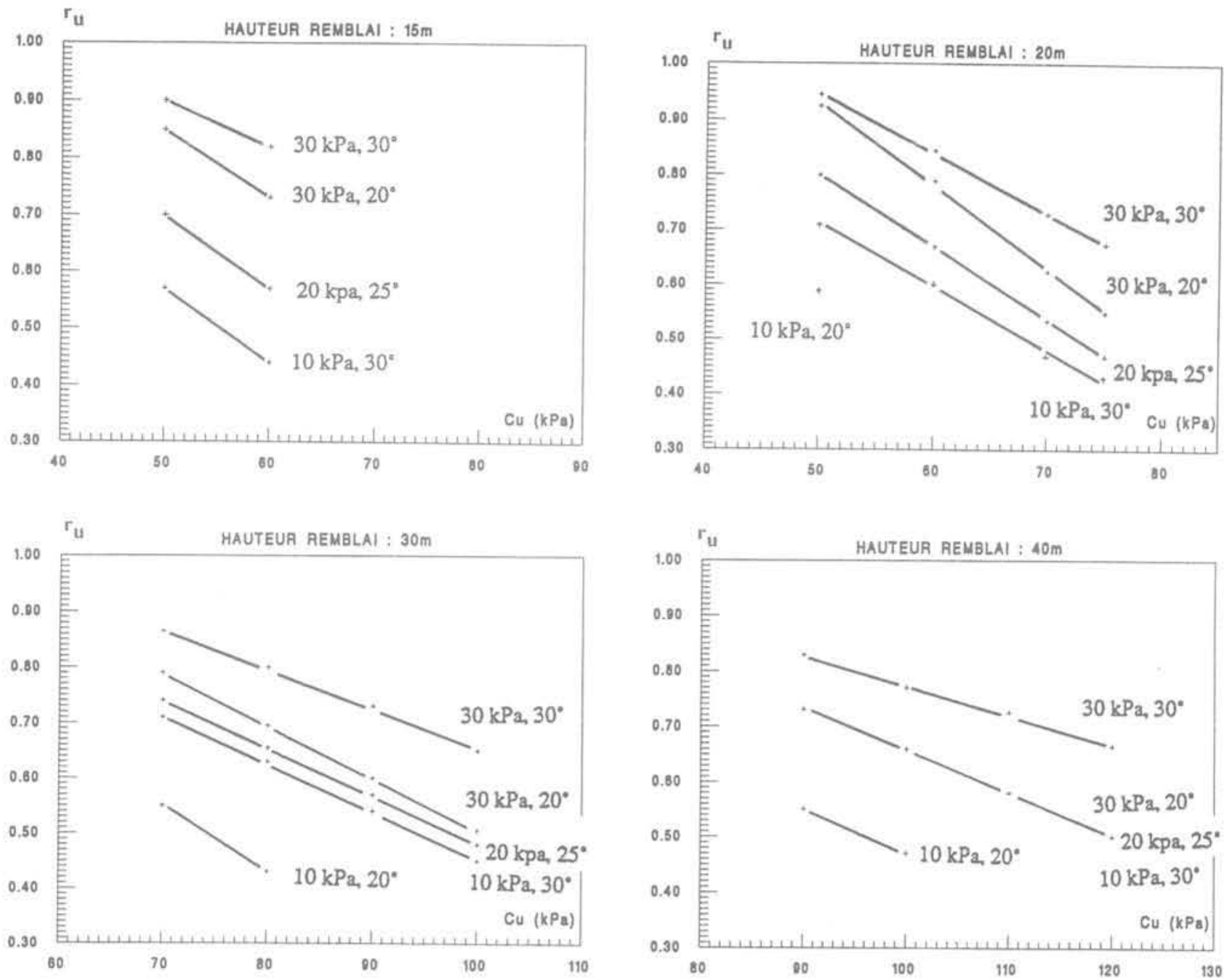

Fig. 14. - Valeurs de $r_{u}$ entrainant $F=1$. - Fig. 14. $-r_{u}$ values leading to $F=1$.

siblement $\mathrm{F}$ lorsque la fondation meuble est peu résistante, le calcul en rupture non circulaire étant généralement le plus défavorable. En ce qui concerne le développement de pressions interstitielles en fin de construction dans un remblai constitué de matériaux fin humides, il est montré que la rupture du talus amont peut intervenir dès que $r_{u}$ atteint 0,6 à 0,7 et celle du talus aval dès que $r_{\text {atteint }} 0,4$ à $0,5, r_{u}$ étant toutefois plus élevé lorsque la pente est définie par une valeur de $c_{\mathrm{u}}$ relativement faible.

\section{BIBLIOGRAPHIE}

(1) ALONSO E., LOUDIERE D., MORLIER P. (1987), Etude des matériaux marneux utilisés pour la construction du barrage de Montbel. Revue Française de Géotechnique, $\mathrm{n}^{\circ} 38,1^{\text {er }}$ trimestre 1987.

(2) BERNEDE T. (1991), Influence des différents paramètres intervenant dans les calculs de stabilité des barrages en terre homogènes. Mémoire du CNAM, Bordeaux, 19 décembre 1991.

(3) COMMISSION INTERNATIONALE DES GRANDS BARRAGES (1986), Calcul statique des barrages en remblai. Bulletin 53, 1986.
(4) FAURE R.M. (1985), Analyse des contraintes dans un talus par la méthode des perturbations. Revue Française de Géotechnique, n³3, 1985.

(5) FREDLUND D.G., KRAHN J. (1977), Comparison of slope stability methods of analysis. Canadian Geotechnical Journal, vol. 14, n 3, 1977.

(6) LEROUEIL S., MAGNAN J.P. TAVENAS F (1985), Remblais sur argiles molles. Technique et Documentation Lavoisier, Paris, 1985.

(7) LONDE P. (1973), La mécanique des roches et les fondations des grands barrages. Commission Internationale des Grands Barrages.

(8) MINISTÈRE DE L'AGRICULTURE (1977), Technique des barrages en aménagement rural.

(9) POULAIN D. (1989), Pressions interstitielles de construction et stabilité des barrages homogènes en matériaux argileux humides. Mémoire de DEA, Université de Bordeaux I, juin 1989.

(10) SMITH G.N. (1990), Elements of Soil Mechanics. BSP Professional Books.

(11) SPENCER E. (1967), A method of analysis of the stability of embankments assuming parallel inter-slice forces. Geotechnique, vol. 17, n 1, 1967. 